\title{
Protective Effect and Possible Mechanisms of Tripterygium Glycosides in Patients with Ankylosing Spondylitis: A Systematic Review and Meta-Analysis
}

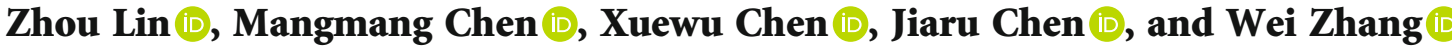 \\ Department of Orthopaedic Surgery, Wenzhou Central Hospital, Wenzhou, Zhejiang 325000, China \\ Correspondence should be addressed to Wei Zhang; wzchopdrzhangwei@126.com
}

Received 11 January 2022; Revised 13 February 2022; Accepted 18 February 2022; Published 3 March 2022

Academic Editor: ChongDe Sun

Copyright (c) 2022 Zhou Lin et al. This is an open access article distributed under the Creative Commons Attribution License, which permits unrestricted use, distribution, and reproduction in any medium, provided the original work is properly cited.

\begin{abstract}
Objective. The safety and efficacy of Tripterygium glycosides (TG) were assessed for ankylosing spondylitis (AS) in accordance with the existing literatures. Materials and Methods. Electronic literature was searched from Chinese VIP databases, Cochrane Library, Chinese Biomedical Literature Database, Wanfang Web of Science, EMBASE, Chinese National Knowledge Infrastructure, and the PubMed for the studies with the publication from the beginning to December 2021. Randomized controlled trials (RCTs) were included only. The major variables of result comprised erythrocyte sedimentation rate (ESR), Creactive protein (CRP), Spinal Pain Visual Analog Score (SP-VAS), Bath Ankylosing Spondylitis Functional Index (BASFI), and Bath Ankylosing Spondylitis Disease Activity Index (BASDAI). Moreover, the secondary variables of result covered the overall clinical effective rate following the adverse drug reaction (ADR). We carried out the meta-analysis with the use of STATA 12.0 and RevMan 5.3. We used GRADE pro3.6.1 software to assess the quality of evidence. Results. In general, we covered 15 randomized controlled trials with the focus of 1186 patients. As proven by our meta-analysis, TG as adjuvant therapy or monotherapy decreased the BASDAI, BASFI, SP-VAS, serum CRP, and ESR than control in patients suffering from AS. Additionally, TG treatment visibly improved the overall effective rate in AS. Nevertheless, TG was not found to significantly increase the rate of ADR in contrast to the control. Conclusion. As indicated by our result, TG may be an option to treat AS. In this paper, we recommended strict trials with high quality and large samples sizes for confirming the finding here.
\end{abstract}

\section{Introduction}

Ankylosing spondylitis (AS) refers to a chronic disease that can cause inflammation. AS can result in damage of structures and inflammation, mostly occurring within the spine or the sacroiliac joint, which normally leads to patients' morning stiffness and chronic back pain and thus causes spinal immobility and ankylosis $[1,2]$. In some severe patients, it ultimately could lead to the rigid and completely fused spine. "Bamboo spine" is formed based on ossification within the fibrous ring's external fibers of intervertebral discs, forming adjoining vertebra's marginal syndesmophyte [3]. Besides, it frequently gives rise to extra-articular aggravation such as inflammatory bowel disease, psoriasis, and uveitis [4]. Normally, young men with 20 to 30 years old are more prone to AS [5]. The estimation of over $90 \%$ genetic role was achieved, the most significant correlation with human leukocyte antigen (HLA)-B27 [6]. Nevertheless, HLA-B27's specific pathogenic effect is still elusive though many hypotheses are proposed.

Currently, AS treatment strategy has been optimized remarkably in past decades by employing tumor necrosis factor- (TNF-) specific agents, disease-modifying antirheumatic drugs (DMARDs), nonsteroidal anti-inflammatory drugs (NSAIDs), and some biologics [7]. Nevertheless, under NSAIDs therapy in the long run, the possible risk covering risk increases in the cardiovascular system, the gastrointestinal tract, and kidney [8]. DMARDs, including sulfasalazine (SSZ) and leflunomide (LEF), have been rarely recommended for treating axial spondyloarthritis, since they have no effect on axial spondyloarthritis, while playing limited roles in treating peripheral manifestations when 
coexisting with the axial disease [9]. Using DMARDs in a long term would have negative effects, for instance, anaphylaxis, gastrointestinal reaction, liver injury, and leukopenia, thus, limiting their use in clinical practice. TNF inhibitor treatment discontinuation within patients usually leads to $84.6 \%$ of partial remission and a relapse within $79.2 \%$, termed responders, in accordance with ASAS40 criterion [10]. Moreover, the huge cost is also a disadvantage of biologics, which should be taken into consideration. As a result, novel therapeutic options or agents are needed for AS treatment.

In recent years, the rising application of alternative and complementary medicine, covering Chinese herbal medicine for AS treatment, arouses wide concern [11, 12]. Tripterygium wilfordii Hook, a woody vine pertaining to the Tripterygium genus, refers to a traditional Chinese medicine having anti-inflammation, antirheumatism, and immunomodulation impacts [13]. Tripterygium glycosides (TG), the main ingredients of Tripterygium wilfordii Hook, were employed in China to treat inflammatory diseases in the long run (e.g., AS, chronic nephritis, a wide variety of skin disorders, and rheumatoid arthritis) [14]. TG can achieve antiinflammatory effect, collateral dredging effect, swelling subsidence effect, detoxification, dampness elimination effect, wind dispelling effect, and inhibition effect on humoral and cell immunity $[15,16]$. TG has been demonstrated with the improvement effect of the clinical characteristics and the regulation effect of AS patients' serum biomarker [17]. Over the past few years, increasing randomized controlled trials with high quality proved TG safety and effectiveness in terms of AS treatment. Nevertheless, there has been rare meta-analysis or systematic review on TG safety and efficacy for AS treatment. For this reason, a systematic review and meta-analysis covering randomized controlled trials with high quality were conducted for assessing TG safety and effect for treating AS.

\section{Methods}

We conducted a systematic review and meta-analysis in accordance with the AMSTAR (assessing the methodological quality of systematic reviews) [18] and PRISMA (Preferred Reporting Items for Systematic Reviews and MetaAnalyses) guidelines. There are no protocols preregistered for this review. This paper was registered in Research Registry (https://www.researchregistry.com/), with reviewregistry1276 as the registration number. Major personal data was not acquired, so we did not need ethical approval. Some methods in this section referred to our previous study [19].

2.1. Database and Searching Strategy. The electronic search was conducted in 8 repositories, i.e., Chinese VIP Database, Cochrane Library, Chinese National Knowledge Infrastructure, EMBASE, Wanfang Database, Web of Science, Chinese Biomedical Literature Database, and PubMed, from their beginning to December 2021. Besides, additional related literatures were manually searched in existing systematic reviews' references. Besides, the searching of existing literatures had no limitation of publication language. The strategy for searching within the English database covered: ([“Trip- terygium wilfordii Hook F”] OR [“Tripterygium wilfordii”] OR ["Tripterygium glycosides"] OR ["Tripterygium"] OR ["thunder god vine"]) AND (["Ankylosing spondylitis"] OR ["AS"]) AND (["random control trials"] OR ["randomized controlled trial"]). For the Chinese databases, we employed free text terms, covering (["lei gong teng (i.e., Tripterygium wilfordii Hook F in Chinese)"] OR ["lei gong teng duo gan (i.e., Tripterygium glycosides in Chinese)"]) AND "qiang zhi xing ji zhu yan (i.e., ankylosing spondylitis in Chinese)" AND "sui ji dui zhao shi yan (i.e., randomized controlled trial in Chinese)". The detailed search strategy used for English databases and Chinese databases was provided as supplementary material (available here).

2.2. Eligibility Standards. The studies were covered in accordance with the PICOS criterion below:

2.2.1. Types of Participants. In this paper, we included patients diagnosed with AS [20] regardless of gender, age, severity, and course of disease.

2.2.2. Types of Interventions. The examined treatment intervention was TG as monotherapy or supplementary treatment with western conventional medication, irrespective of the therapy's administration period, administrated methods, administration route, duration, or dosage.

2.2.3. Types of Controls. The control was given western conventional medication. We excluded studies involving controls of Chinese herbal medicine treatments.

2.2.4. Types of Result Measures. The primary outcome measures covered (1) SP-VAS (Spinal Pain Visual Analog Score), (2) BASFI (Bath Ankylosing Spondylitis Functional Index), (3) BASDAI (Bath Ankylosing Spondylitis Disease Activity Index), (4) ESR (erythrocyte sedimentation rate), and (5) CRP (C-reactive protein). ADR (adverse drug reaction) and the clinical effective rate (ER) were the secondary outcomes.

2.2.5. Types of Studies. We only involved randomized controlled trials investigating the safety and efficacy of Tripterygium glycosides (TG) for AS with no limit of publication states or languages. If this paper searched an article that contained 3 treatment arms, we merely extracted data for the control arm (s) as well as the arm (s) that entails TG. Quasi-randomized trials with researches allocating subjects were excluded according to the admission number order and the date of birth.

2.3. Exclusion Standards. (1) Nonrandomized controlled trial; (2) randomized controlled trials in which the patients were not reported to suffer from AS; (3) the combination of TG treatment and other drugs; (4) consistent animal experiments, reviews, and abstracts; (5) the duplication of publications and randomized controlled trials containing not sufficient data.

2.4. Literature Selection. In this paper, we used the flow diagram of PRISMA to choose the involved articles. The result of literature was introduced to the Endnote X7 software. 2 
authors evaluated the potential eligible studies independently after they screened the title and abstract for the removal of duplication and not relevant research or the randomized controlled trials that were not involved in the inclusion standards. Afterward, we obtained the full text of the rest potential researches and reviewed these researches. A third independent investigator would resolve the disagreement between the first 2 authors.

2.5. Data Abstraction. 2 reviewers searched the data independently. Next, the uniformity was examined by a third independent reviewer. We used a standard form containing the retrieved items, which covered the general information of studies: the author's (s) name(s), publication date, design of study, patient gender and age, number of sample, intervention approach covering TG alone, or supplement western conventional medication and course of treatment. For continuous outcomes, we extracted mean and standard deviation (SD) as well as participant number of each research. For dichotomous outcomes, the total number and the number of events of both groups were extracted. Under probable conditions, we recalculated the data according to other types of form, as an attempt to carry out pooled analysis. Discussions would be conducted to solve the disagreement of the mentioned 2 reviewers. If necessary, we established contact with the involved studies' authors to acquire the missing data or additional data.

2.6. Evaluation of the Quality of Included Studies. With the use of the Cochrane collaboration tool, 2 authors independently evaluated the method quality and the bias risk in the included randomized controlled trial studies [21]. The above Cochrane tool is capable of evaluating incomplete result data, selective result reporting, result evaluation blinding, blinding of subjects, allocation concealment, randomization, and other types of biases in terms of the respective item. It can also classify studies into high risk of bias, low risk of bias, or not clear level of bias.

2.7. Evidence Quality Assessment. We evaluated the quality of the evidence according to the GRADE [22, 23]. We classified the meta-analysis results into high evidence quality, moderate evidence quality, low evidence quality, or very low evidence quality. First, we classified the randomized controlled trial results into evidence with high quality. The quality of each result decreased due to indirectness, publication bias, inconsistency, imprecision, and risk of bias. We adopted GRADE pro3.6.1 for the investigation and synthesis of data.

2.8. Statistical Analysis. The data were collected and input into the STATA software (V.12.0; StataCorp, College Station, TX) for meta-analysis. The bias risk assessment was evaluated by RevMan software version 5.3 (Cochrane Collaboration, Oxford, UK). For continuous outcomes, mean, standard deviation (SD), and sample number of each group were extracted. For dichotomous outcomes, the total number and the number of events of both groups were extracted. Standard mean difference (SMD) with $95 \%$ confidence interval (CI) was calculated to analyze continuous variables (ESR,
CRP, SP-VAS, BASFI, and BASDAI). Relative risk (RR) with 95\% CI was calculated for dichotomous variables (overall effective rate and ADR). Statistical heterogeneity among trials was assessed using the chi-squared test and $I^{2}$ statistic. The heterogeneity among studies was identified if the $I^{2}$ was greater than or equal to $50 \%$ or $P$ value was less than or equal to 0.05 . A random-effects model was applied when heterogeneity was detected or the statistical heterogeneity was high $\left(P<0.05\right.$ or $\left.I^{2}>50 \%\right)$, and then, further subgroup study and meta-regression analysis (the number of included studies was more than 8) were performed to detect the origin of heterogeneity. Otherwise, a fixed-effects model was used $\left(P \geq 0.05\right.$ or $\left.I^{2} \leq 50 \%\right)$. Publication bias was assessed by using Begg's and Egger's linear regression test in the metaanalysis, and we considered $P<0.05$ to be significant. Stabilities of synthetic results were evaluated with a visual assessment of sensitivity analyses. The method of omitting each study in sequence was used for sensitivity analysis.

\section{Result}

3.1. Study Description. We found 320 related studies in the databases searched. Afterward, 312 studies were left when duplicate removal was achieved. Subsequently, we removed 285 studies based on specific tittle and abstract screening. By screening 27 articles' full texts, we removed 12 studies due to the nonconformity with the study inclusion standards. Finally, 15 studies [24-38] were involved for investigation. Figure 1 presents the search standards and the process of selection specifically.

3.2. General Characteristics of the Covered Studies. Table 1 lists the characteristic exhibited by all the included randomized controlled trials. The publication of the trails ranged from 2011 to 2021. In general, the randomized controlled trials covered 1186 patients, 569 within the control in comparison with 617 within the experiment group. The studies generally tested the impact arising from TG on AS, 8 studies $[25,31-34,36-38]$ used sulfasalazine as the control, 3 studies $[24,29,35]$ used leflunomide as the control, and 4 studies $[26-28,30]$ used etanercept as the control. The researches covered no less than 8 weeks of the intervention with the exception of 2 studies $[32,35]$. They did not report the course of treatment in their studies.

3.3. Risk of Bias. In this paper, we applied the Cochrane risk of bias tool for exploring the bias risk. According to the 15 included studies, the standard number had a range ( $4 / 7$ to 6/7). Besides, 10 of the included randomized controlled trials $[24,25,27-29,31,32,34,36,38]$ presented the precise method used for generating random sequence. 10 studies $[25-27,30,31,33,35-38]$ indicated the blinding, and the rest were still not clear. 10 studies $[25,27-29,32-35,37$, 38] complied with the not complete result data standards because no drop-out patient or drop-out information was found. 2 studies [36, 37] contained bias risk within selective reporting. A baseline comparison was drawn, and the participants' consent was effectively documented. Furthermore, most studies included did not show other biases. More 


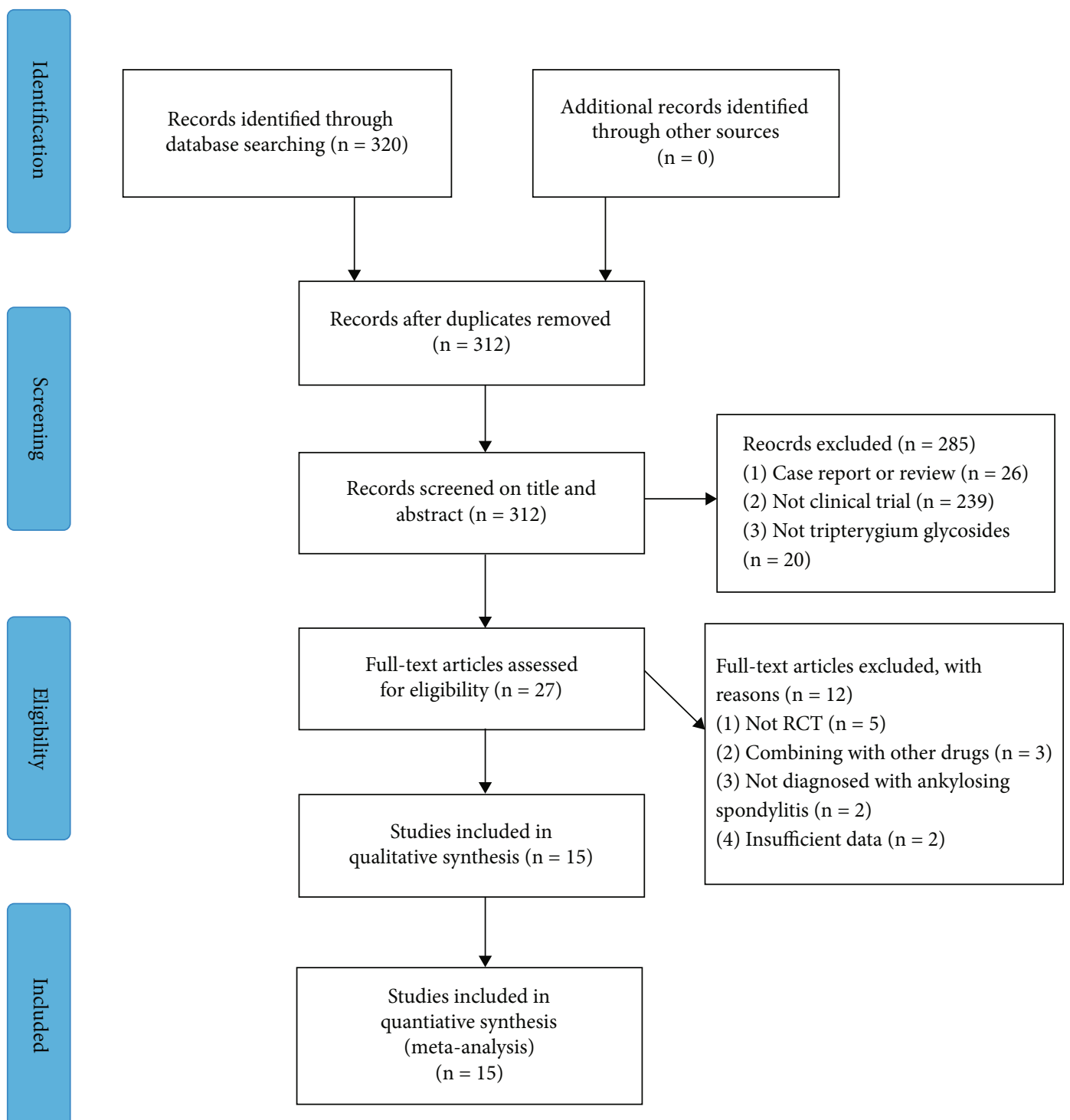

Figure 1: Flowchart of study selection.

specific information regarding risk of bias assessment of the respective trial is presented in Figures 2 and 3.

\subsection{Results of Meta-Analysis}

3.4.1. BASDAI. 4 studies [24, 29, 36, 37] compared TG alone with the control regarding BASDAI. According to Figure 4(a), as revealed by the pooled result, TG monotherapy was remarkable for reducing BASDAI in contrast to the control $(\mathrm{SMD}=-1.116 ; 95 \% \mathrm{CI}=-2.001$ to $-0.232 ; P=$ 0.013 ; heterogeneity $\chi^{2}=33.97, \mathrm{df}=3, I^{2}=91.2 \%, \quad P<$ 0.001). 4 studies $[26,30,34,38]$ reported TG plus control versus control in accordance with BASDAI. The pooled results illustrated that TG plus control had significance in terms of the reduction of BASDAI in contrast to the control $(\mathrm{SMD}=-1.578 ; 95 \% \mathrm{CI}=-1.940$ to $-1.216 ; P<0.001$, heterogeneity $\quad \chi^{2}=6.37, \quad \mathrm{df}=3, \quad I^{2}=52.9 \%, \quad P=0.095$, Figure 4(b)). Meta-regression was used for the exploration of heterogeneity sources. To find the probable source of het- erogeneity in studies, we carried out the meta-regression investigation in terms of sample size, year of publication, age, and treatment course (Figure 5). Overall, the course of treatment $\left(\beta=-0.069 ; P=0.04\right.$; Adj $\left.R^{2}=42.53 \%\right)$ might be the major heterogeneity source. However, the sample size $\left(\beta=0.015 ; P=0.302 ;\right.$ Adj $\left.R^{2}=3.66 \%\right)$, age $(\beta=0.087 ; P=$ $\left.0.285 ; \operatorname{Adj} R^{2}=3.52 \%\right)$, and publication year $(\beta=-0.107 ; P$ $\left.=0.238 ; \mathrm{Adj} R^{2}=14.81 \%\right)$ were not the prominent heterogeneity sources for BASDAI.

3.4.2. BASFI. 2 studies [24, 37] drew the comparison between TG alone and the control for BASFI. The available data illustrated that TG monotherapy dramatically depressed the BASFI in contrast to the control $(\mathrm{SMD}=-1.463 ; 95 \% \mathrm{CI}=-1.794$ to $-1.131 ; P<0.001$; het erogeneity $\quad \chi^{2}=0.10, \quad \mathrm{df}=1, \quad I^{2}=0 \%, \quad P=0.751$, Figure 6(a)). 5 studies [26, 30, 31, 34, 38] drew the comparison between TG plus control and the control about BASFI. 


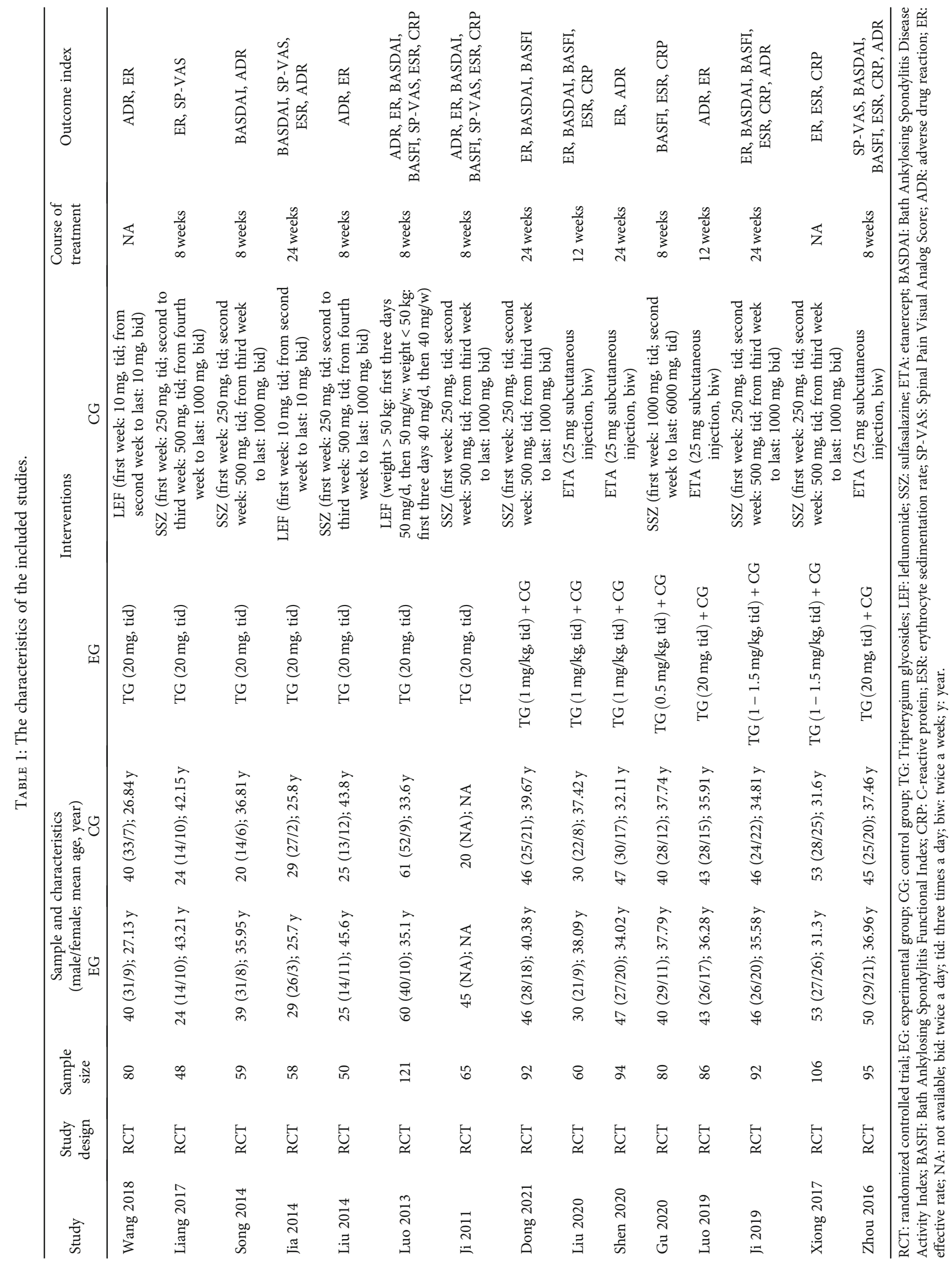




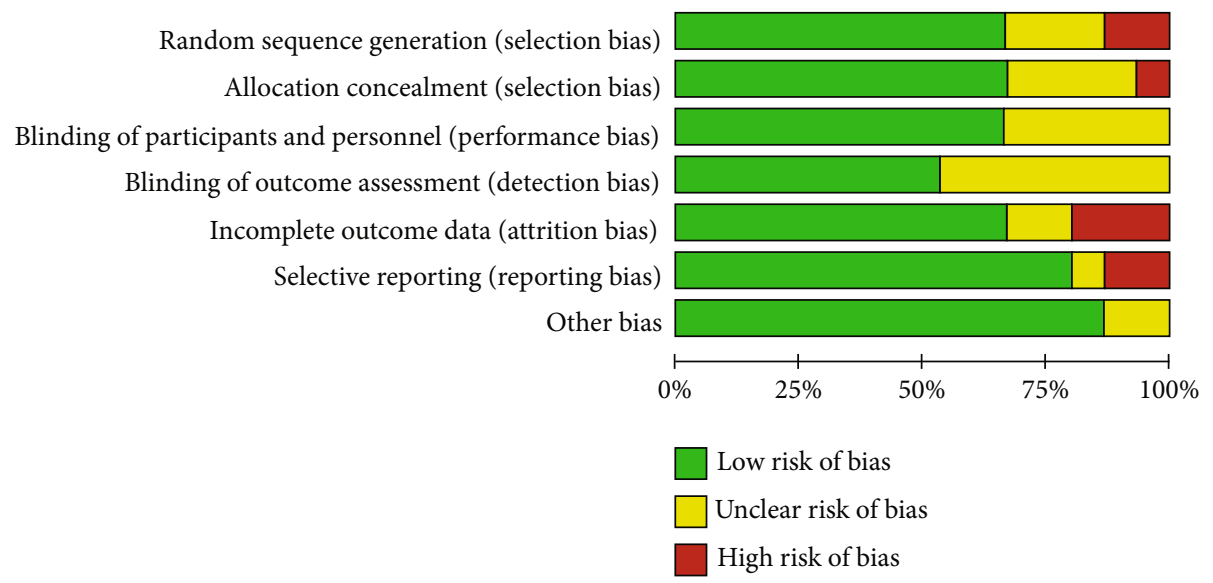

FIGURE 2: Risk of bias graph: review authors' judgements about each risk of bias item presented as percentages across all included studies.

As revealed by the pooled result, TG and western medicine combination noticeably reduced BASFI in contrast to western medicine alone $(\mathrm{SMD}=-1.781 ; 95 \% \mathrm{CI}=-2.008$ to $-1.553 ; P<0.001$, heterogeneity $\chi^{2}=2.18$, df $=4, I^{2}=0 \%$, $P=0.702$, Figure 6(b)).

3.4.3. SP-VAS. 4 studies $[24,25,29,37]$ reported TG alone versus control in accordance with SP-VAS. According to the pooled results, TG monotherapy had significance in terms of lessening SP-VAS in contrast to the control $(\mathrm{SMD}=-0.970 ; 95 \% \mathrm{CI}=-1.793$ to $-0.147 ; P=0.021$, heterogeneity $\chi^{2}=29.63, \quad \mathrm{df}=3, \quad I^{2}=89.9 \%, \quad P<0.001$, Figure 7(a)). Only one study [26] compared TG plus control with the control for SP-VAS. As revealed by the study, TG and western medicine combination significantly reduced SP-VAS in comparison with western medicine alone $(\mathrm{SMD}=-1.344 ; \quad 95 \% \mathrm{CI}=-1.791$ to $-0.898 ; \quad P<0.001$, Figure $7(\mathrm{~b}))$.

3.4.4. CRP. There were 2 studies $[24,37]$ that compared TG plus control with the control about CRP. As revealed by the pooled result, TG monotherapy markedly downregulated CRP level in contrast to the control $(\mathrm{SMD}=-0.492 ; 95 \%$ $\mathrm{CI}=-0.792$ to $-0.193 ; P=0.001$, heterogeneity $\chi^{2}<0.001$, $\mathrm{df}=1, I^{2}=0 \%, P=0.959$, Figure $\left.8(\mathrm{a})\right) .5$ studies $[26$, $30-32,38]$ referred to TG plus control versus control on CRP. According to the pooled data, TG plus control distinctly decreased $\mathrm{CRP}$ in contrast to the control $(\mathrm{SMD}=-0.890 ; 95 \% \mathrm{CI}=-1.088$ to $-0.692 ; P<0.001$, heterogeneity $\chi^{2}=2.55$, df $=4, I^{2}=0 \%, P=0.636$, Figure $8(\mathrm{~b})$ ).

3.4.5. ESR. 3 studies $[24,29,37]$ compared TG alone with the control with regards to ESR. According to Figure 9(a), the pooled results exhibited that TG monotherapy was noticeable in lowering ESR in contrast to the control $(\mathrm{SMD}=-0.331 ; 95 \% \mathrm{CI}=-0.588$ to $-0.073 ; P=0.012$; heterogeneity $\left.\chi^{2}=0.06, \mathrm{df}=2, I^{2}=0 \%, P=0.972\right) .5$ studies $[26,30-32,38]$ mentioned TG plus control versus control about ESR. As revealed by the pooled data, TG plus control notably reduced ESR in contrast to the control $(\mathrm{SMD}=-1.307 ; 95 \% \mathrm{CI}=-1.515$ to $-1.098 ; P<0.001$, het- erogeneity $\quad \chi^{2}=4.93, \quad \mathrm{df}=4, \quad I^{2}=18.9 \%, \quad P=0.294$, Figure 9(b)).

3.4.6. ER. There were 5 studies [24, 25, 33, 35, 37] comparing TG alone with the control about the effective rate. According to the pooled data, TG monotherapy evidently elevated effective rate in contrast to the control $(\mathrm{RR}=1.295 ; 95 \% \mathrm{CI}$ $=1.112$ to $1.509 ; P=0.001$, heterogeneity $\chi^{2}=3.40, \mathrm{df}=4$, $I^{2}=0 \%, P=0.493$, Figure $\left.10(\mathrm{a})\right) .6$ studies $[27,28,30,32$, 34,38 ] referred to TG plus control versus control for the effective rate. The pooled data revealed that TG plus control obviously enhanced effective rate in comparison to the control $(\mathrm{RR}=1.247 ; 95 \% \mathrm{CI}=1.150$ to $1.353 ; P<0.001$, heterogeneity $\chi^{2}=0.19$, df $=5, I^{2}=0 \%, P=0.999$, Figure $10(\mathrm{~b})$ ).

3.4.7. $A D R$. In this paper, we found ADR from 9 studies [26-29, 33, 35-38]. The adverse event frequency reached $61 / 364$ of the control and 45/315 of the trial group. According to the pooled data, the ADR rate reported insignificant difference in the 2 groups $(\mathrm{RR}=1.216 ; 95 \% \mathrm{CI}=0.848$ to 1.742; $P=0.288$, heterogeneity $\chi^{2}=12.28, \mathrm{df}=8, I^{2}=34.9$ $\%, P=0.139$, Figure 11). As revealed by the results of this paper, amenorrhea, menstruation disorders, and liver function damage or gastrointestinal discomfort frequently constitute the most frequently occurring adverse events. Significant negative effects were mild, without any high negative effect, covering life threatening indicated by the covered randomized controlled trials.

3.5. Subgroup Analysis. We carried out subgroup investigation in accordance with the course of treatment, sample size, age, control medication, and TG dosage in Table 2 because of the significant heterogeneity of SP-VAS and BASDAI result in our paper. Nevertheless, according to the results of subgroup investigation, the mentioned factors were not the prominent heterogeneity source in terms of BASDAI and SP-VAS.

3.6. Publication Bias and Sensitivity Investigation. In this paper, we performed the Begg's test and Egger's test (Figure 12) for examining ADR's possible publication bias within this meta-analysis. Consequently, according to the $P$ 


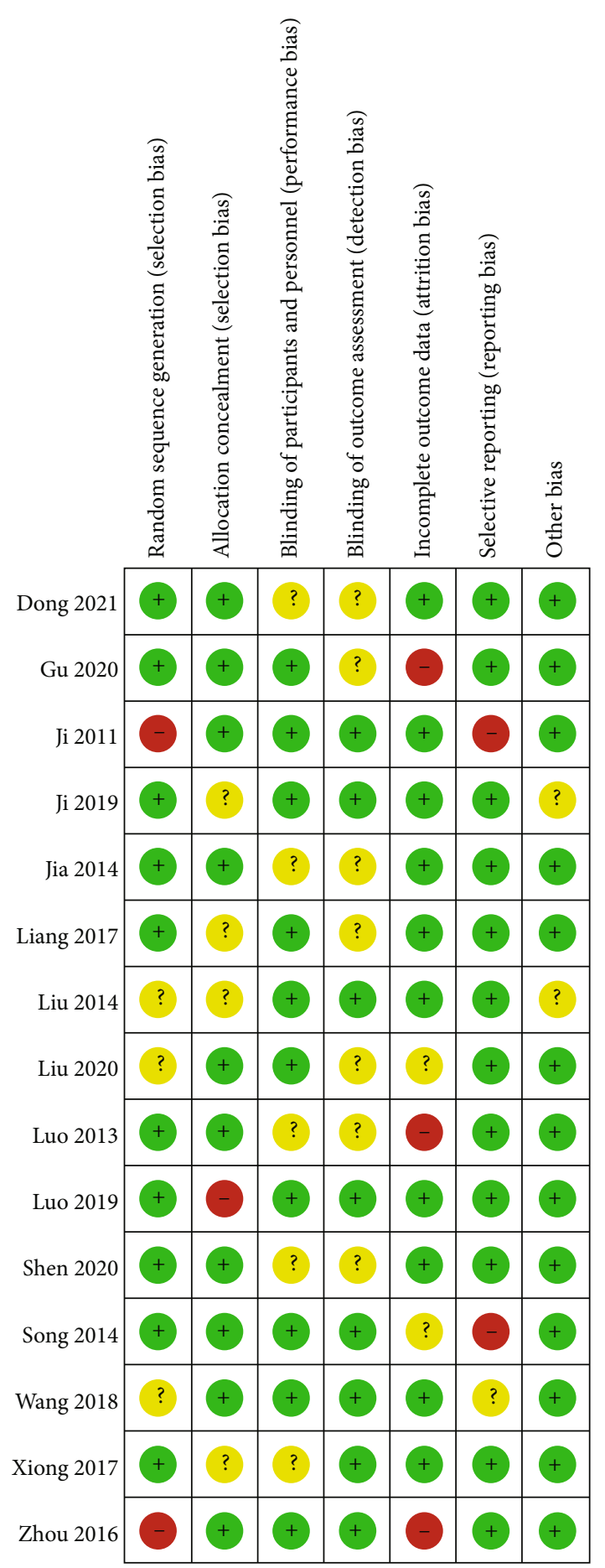

Figure 3: Risk of bias summary: review authors' judgements about each risk of bias item for each included study.

values from Begg's test and Egger's test, ADR had no remarkable publication bias $(P=0.251$ and $P=0.330$, separately).

For establishing the impact arising from each involved studies relating to BASDAI, BASFI, CRP, ESR, ADR, and ER pooled data to demonstrate the findings here to be robust, the sensitivity was evaluated through the exclusion of one study at a time and computing the pooled data for other randomized controlled trials. According to the sensitivity investigation results, no noticeable effect was exerted on pooled data when the respective study was eliminated, respectively, thereby indicating the finding here to be relatively robust (Figure 13).

3.7. GRADE Assessment. We used the GRADE method for exploring the results' evidence quality, with moderate or low methodological issues and heterogeneity, as well as significantly low quality. According to Table 3, the evidence quality of 5 results was low, one result had very low evidence quality, and another result had moderate evidence quality. Thus, the overall evidence quality of this study was low.

\section{Discussion}

AS, the axial skeleton's chronic inflammatory disease, leads to the rigid and completely fused spine [39, 40]. Medications (NSAIDs and DMARDs) used to treat AS have been used in clinical practice for many years [8]. However, the diverse adverse reaction such as anaphylaxis, gastrointestinal reaction, liver injury, and leukopenia limit their longterm use. Tumor necrosis factor blockers refer to a promising drug to treat AS. Nevertheless, tumor necrosis factor blockers are likely to lead to some significant side effects, covering neurological issues and reactivating latent tuberculosis [41]. Therefore, safe and effective anti-AS drugs are urgently required. With the progress of clinical practice and research, increasing evidence reveals that Chinese herbal medicine has prominent effects in preventing and treating AS [14]. TG, extracted from Traditional Chinese Medicinal plant Tripterygium, has been used in China and other Asian countries for the long-term treatment of inflammatory diseases covering AS, chronic nephritis, different skin disorders, and rheumatoid arthritis [15]. According to modern pharmacological research, TG can achieve anti-inflammatory effect, collateral dredging effect, swelling subsidence effect, detoxification, dampness elimination effect, wind dispelling effect, and inhibition effect on humoral and cell immunity $[15,16]$. Considerable randomized controlled trials have demonstrated that TG was advantageous in treating AS [27, 31, 34], whereas no high-quality systematic review and meta-analysis with large sample size on the safety and efficacy of TG to treat AS has been demonstrated. Thus, a systematic review and meta-analysis was carried out for the investigation of whether TG has safety and efficacy in patients suffering from AS to evidence clinical practice and scientific study.

4.1. Summary of Evidence. The current paper has been the initial systematic review and meta-analysis investigating whether TG monotherapy and TG supplied with western medicine have efficacy and safety to treat AS. On the whole, fifteen high-quality randomized controlled trials involving 1186 individuals with AS were included in the investigation. According to the major finding of existing systematic reviews and meta-analyses, using TG as adjuvant therapy or monotherapy for AS treatment evidently decreased BASDAI, BASFI, and SP-VAS, thereby demonstrating that TG 


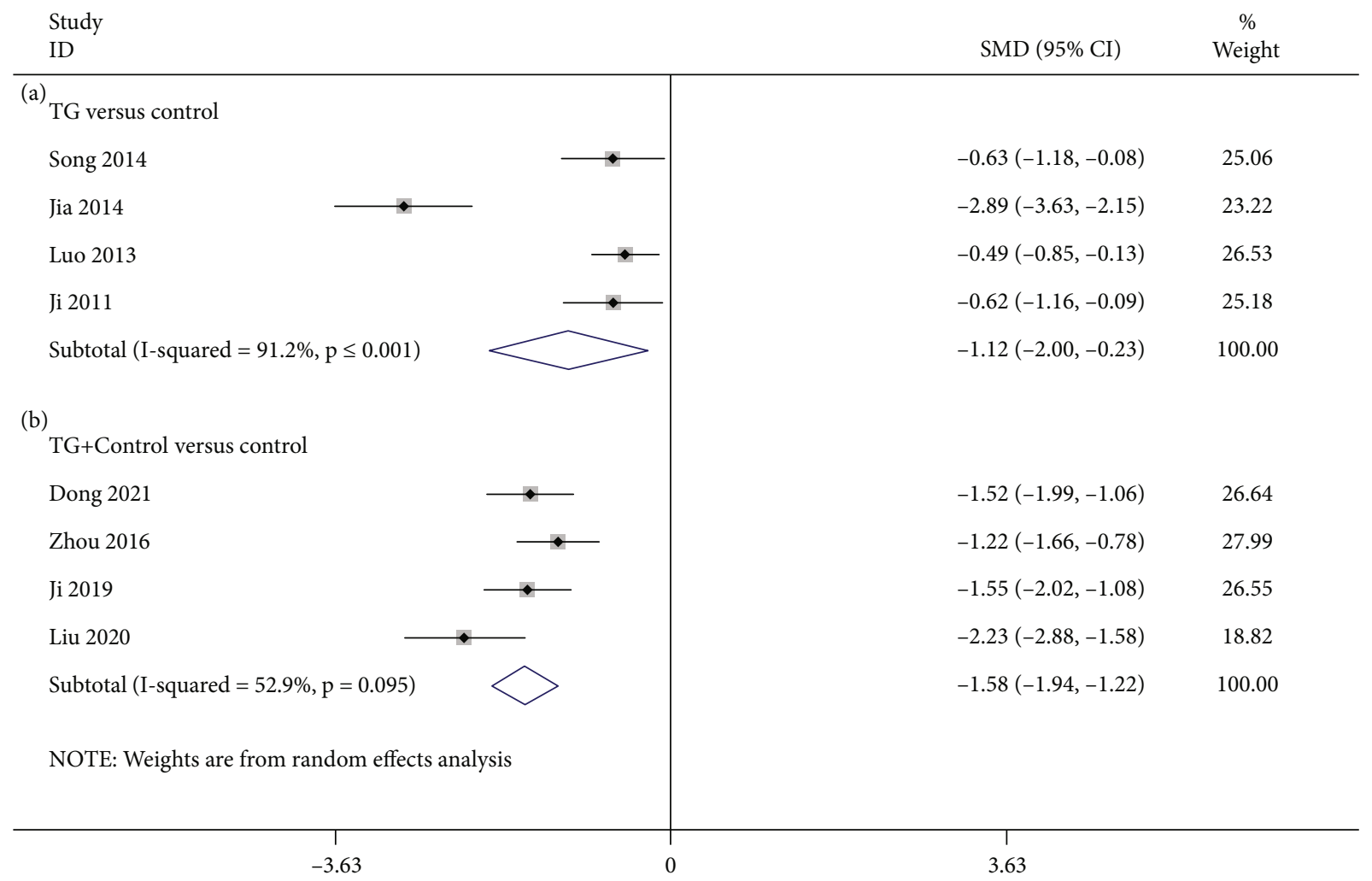

Figure 4: Forest plot of BASDAI: (a) TG versus control and (b) TG plus control versus control.

can effectively relieve the pain that is caused by AS. Next, the serum CRP and ESR were lowered by TG. In addition, TG treatment visibly elevated the overall effective rate in AS. Yet, there was no significant difference in the incidence of adverse reactions between the 2 groups, thereby demonstrating that TG has good safety and tolerability for AS patients. Thus, supporting evidence was provided in our paper, showing that TG may be highly recommended for planned use in patients suffering from AS.

4.2. Comparison with Existing Studies. Some systematic reviews and meta-analyses demonstrated TG's efficacy and safety in AS treatment. As revealed by a meta-analysis [42] constituting 14 randomized controlled trials involving 996 patients, total effective rate, BASDAI, BASFI, and CRP showed no significant difference between the TG group and the western medicine group, inconsistent with the results of this paper. In their study, only 6 studies comparing TG with western medicine, and the rest 8 studies were compared with Chinese herbal compound. However, the studies with Chinese herbal compound control were excluded because numerous varieties of Chinese herbal compound of the control may result in high heterogeneity, and only the studies with western medicine control were involved. Thus, the sample size of this paper was significantly larger than theirs, which may be major cause of contradiction. Besides, part of the finding here was consistent with the data of Li et al. [3]. They performed a meta-analysis involving 11 trials that focused on 807 patients and exhibited that TG treatment obviously decreased the pain index, ESR in AS.
Nevertheless, the CRP was not reduced in their study. The data here have no consistency with the findings of existing researches in several fields, probably correlated with (1) the included studies in the existing meta-analyses varied considerably in quality. Nonetheless, herein, randomized controlled trials having a risk of bias score $\geq 4$ on the basis of the Cochrane RoB tool were included, implying that only high-quality randomized controlled trials were included in this paper. (2) The diversity and complexity of Chinese herbal compound in the control in the existing studies are also major causes of the difference. Nevertheless, herein, we focused particularly on the single western medicine in the control, which can reduce the risk of heterogeneity. (3) AS is a chronic condition with diverse stages. The AS distinct stages can affect the progress of the disease along with the treatment response. However, many existing studies and this paper have not reported the stages of AS, which may also lead to contradictions. (4) The prevalence of AS in young men is 10 times higher than that in women. Therefore, the gender ratio difference between existing studies and this paper also affects the research results. (5) The course of treatment may affect the outcome. The duration of the current and current studies was $8-24$ weeks. It is a paradox that too short a course of treatment may lead to poor efficacy, while too long a course of treatment may lead to serious adverse reactions.

4.3. Strengths. The advantages of our meta-analysis studies include a clearly defined study issue, namely, reduced selection of randomized controlled trials with consistency, 


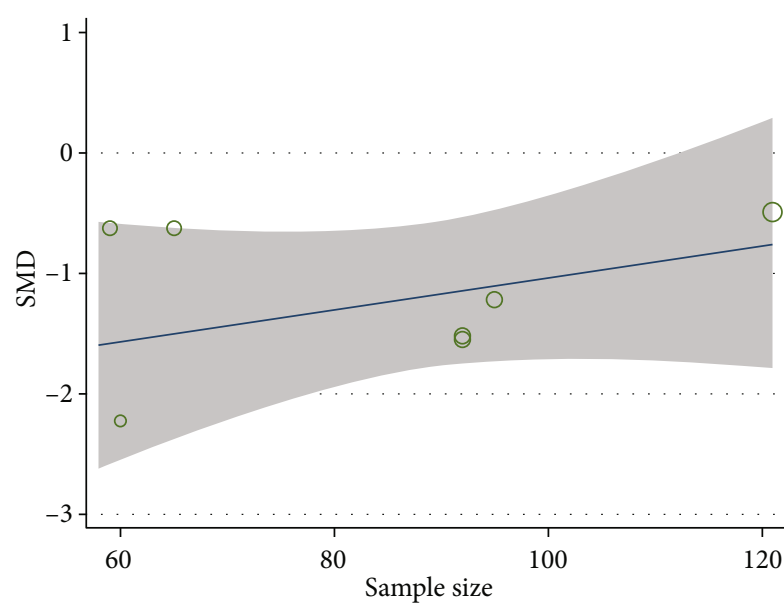

(a)

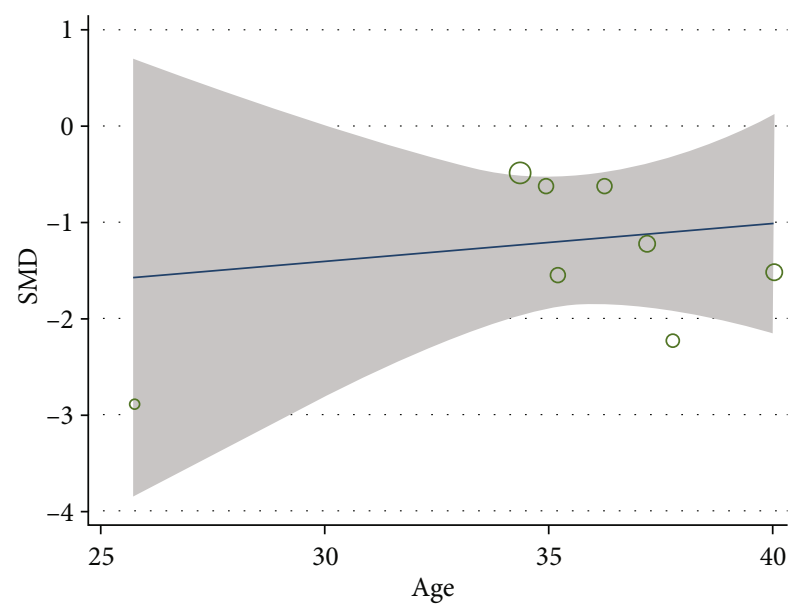

(c)

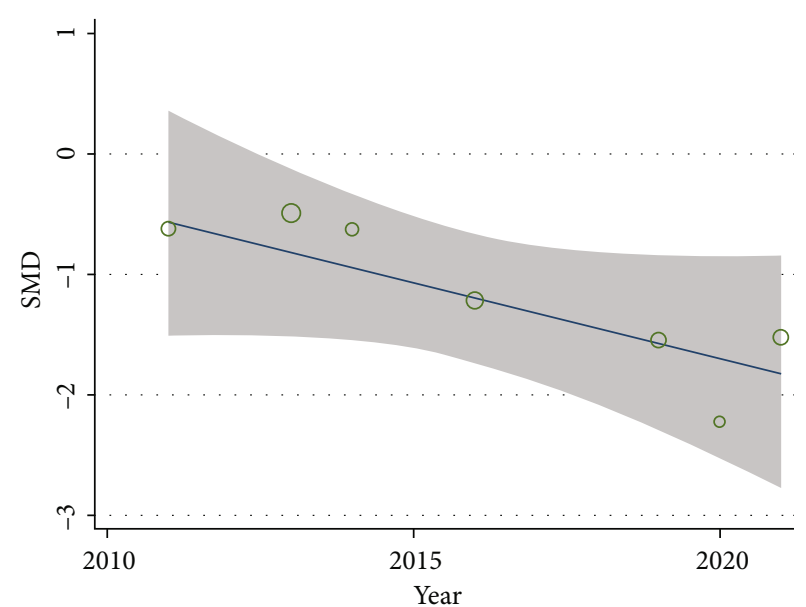

(b)

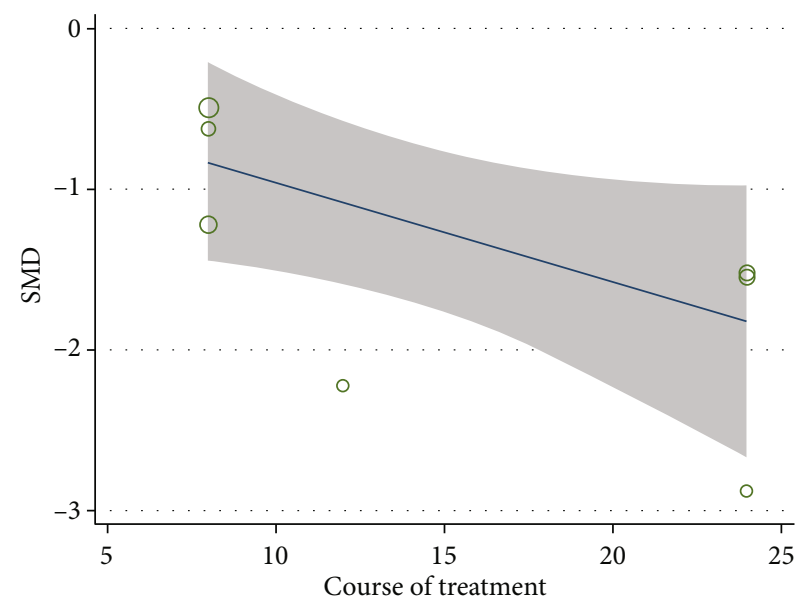

(d)

Figure 5: Meta-regression analysis of BASDAI. (a) Sample size. (b) Publication year. (c) Age. (d) Course of treatment.

fidelity, and bias. A precise study methodology was designed prior to the meta-analysis and an in-depth search of the literature was conducted. 2 researchers evaluated the protocols for the input data components, as well as the quality control of all data. All of the studies covered in herein constituted randomized controlled trials with an extraordinary number being high quality, which contributes to overcoming the drawbacks of the recall or selection bias in terms of nonrandomized researches. In addition, the number of trials and overall sample size was large (15 trials, 1186 participants). Subgroup and meta-regression assessments were carried out for determining the source of heterogeneity. Therefore, no publication bias was reported in this meta-analysis, and sensitivity estimates suggest that the results of the present meta-analysis have relative robustness.

4.4. Limitations. However, this paper had some limitations. First, no protocol has been preregistered for our paper, probably leading to potential bias to our paper. Second, although randomized controlled trials were covered, the covered major studies had some intrinsic and methodological shortcomings: (1) only 10 trials reported information generated by randomization. (2) Some studies did not report the blinded process or had unclear results, which might lead to unintentional or intentional biases to their results and reduce the credibility of their research conclusions. Three blinds are required in further trials. Third, AS is a chronic disease that requires lifelong treatment. The long-term safety and efficacy of drugs are the key to determine the clinical efficacy of therapeutic drugs. However, the treatment period of our paper was between 8 and 24 weeks. The long-term safety of TG was not found for AS because the duration of treatment of the covered studies was short, and no dropouts were revealed in a considerable number of the covered studies. Fourth, the dosage, administration methods, and course of TG treatments differed remarkably in the major randomized controlled trials. This clinical heterogeneousness might jeopardize the feasibility of the results of this paper. Fifth, we searched researches published in Chinese or English repositories only, so the potentially relevant randomized controlled trials published in other languages might be excluded. Besides, all randomized controlled trials covered here were made in China, a potential limitation to the generalizability of the results of this paper. Sixth, our paper had low quality of evidence due to the high risk of bias and inconsistency. Therefore, it is necessary to further carry out 


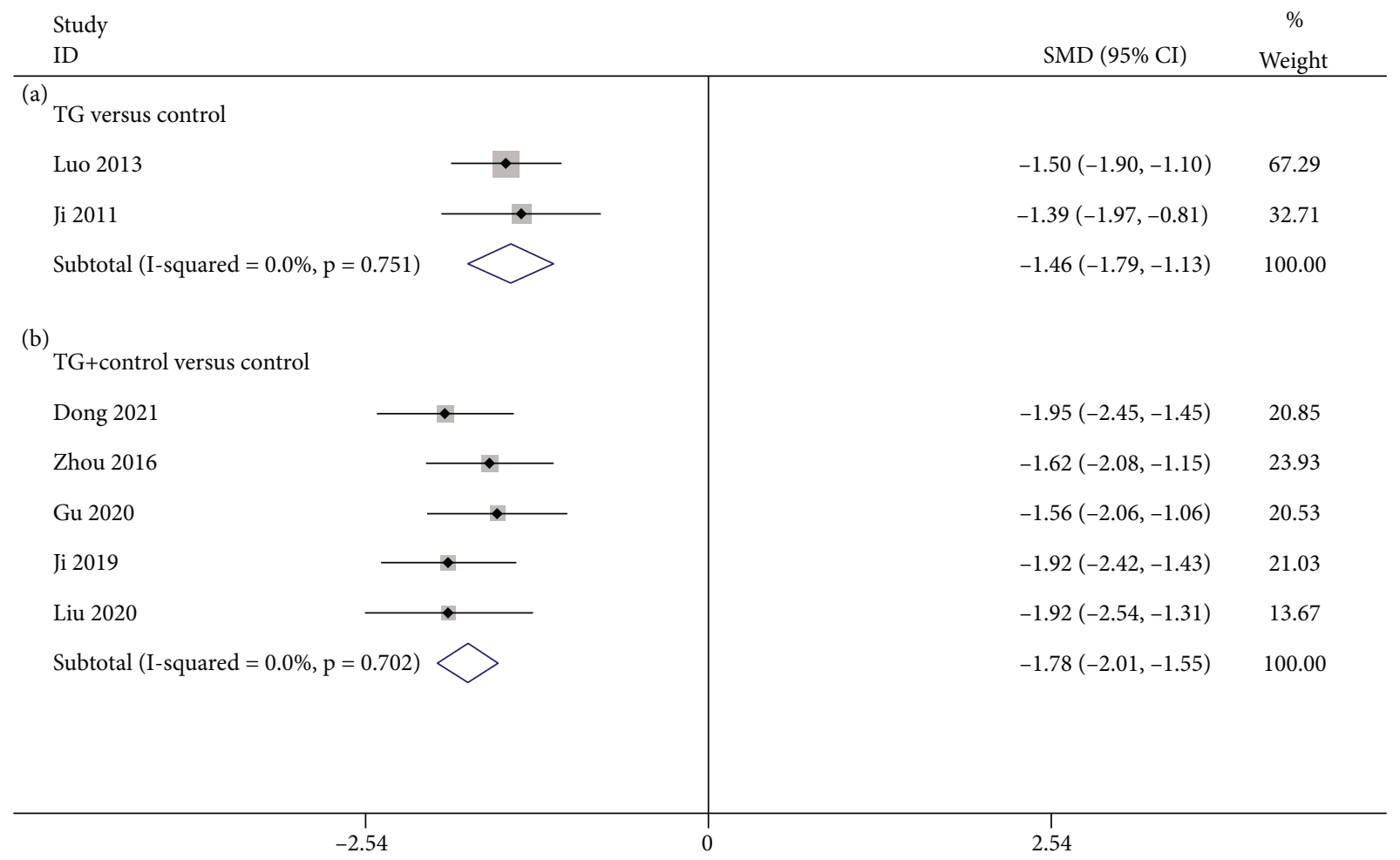

FIgUre 6: Forest plot of BASFI: (a) TG versus control and (b) TG plus control versus control.

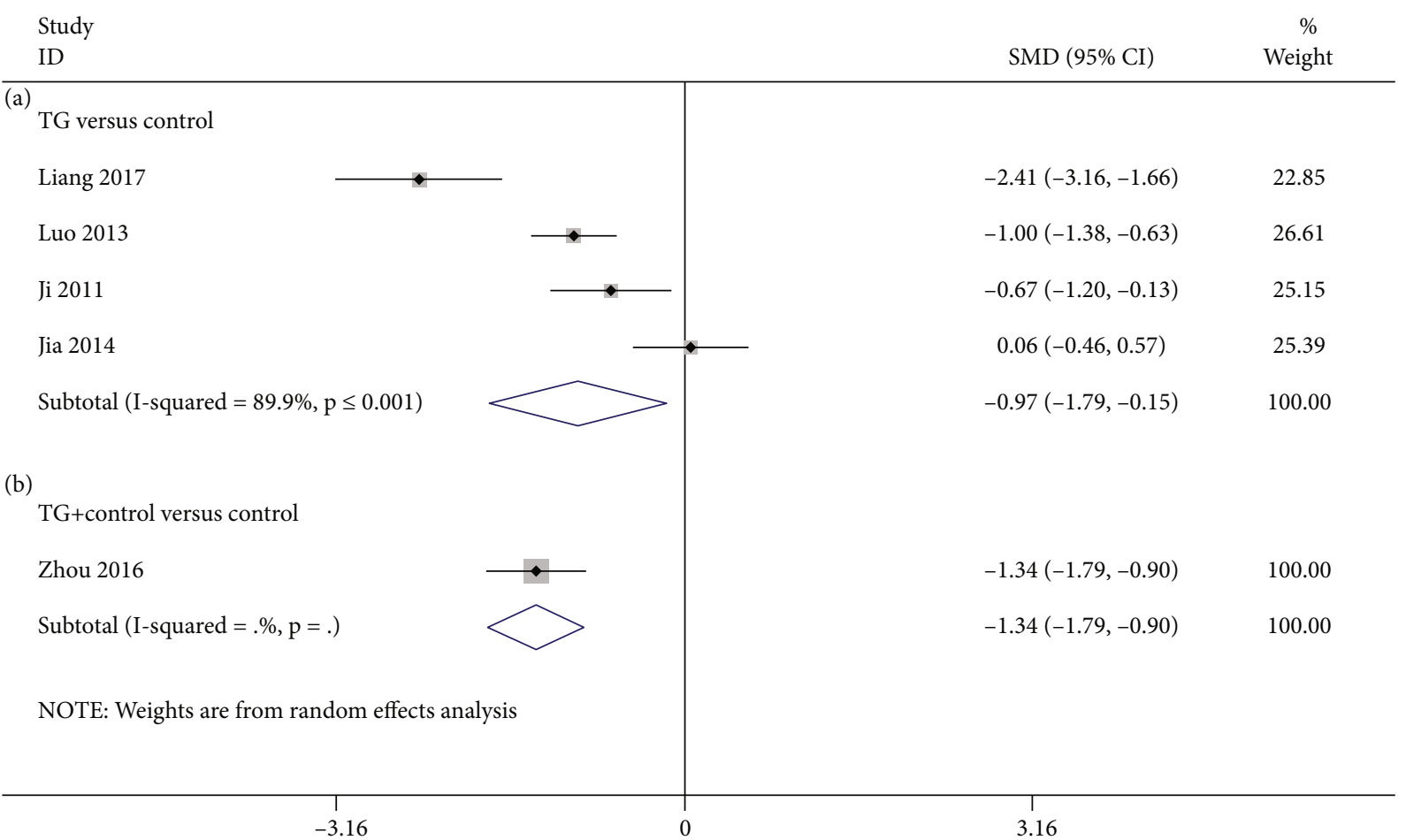

FIgUre 7: Forest plot of SP-VAS: (a) TG versus control and (b) TG plus control versus control.

multicenter randomized controlled trials of high-quality TG in the treatment of AS worldwide, so the data can provide a worldwide reference.
4.5. Implications for Research. Important ideas that may advance research in this area are revealed here. First, strategies to improve the methodological quality of randomized 


\begin{tabular}{l} 
Study \\
ID \\
\hline (a) SMD versus control $(95 \%$ CI $)$
\end{tabular}

FIgURE 8: Forest plot of CRP: (a) TG versus control and (b) TG plus control versus control.

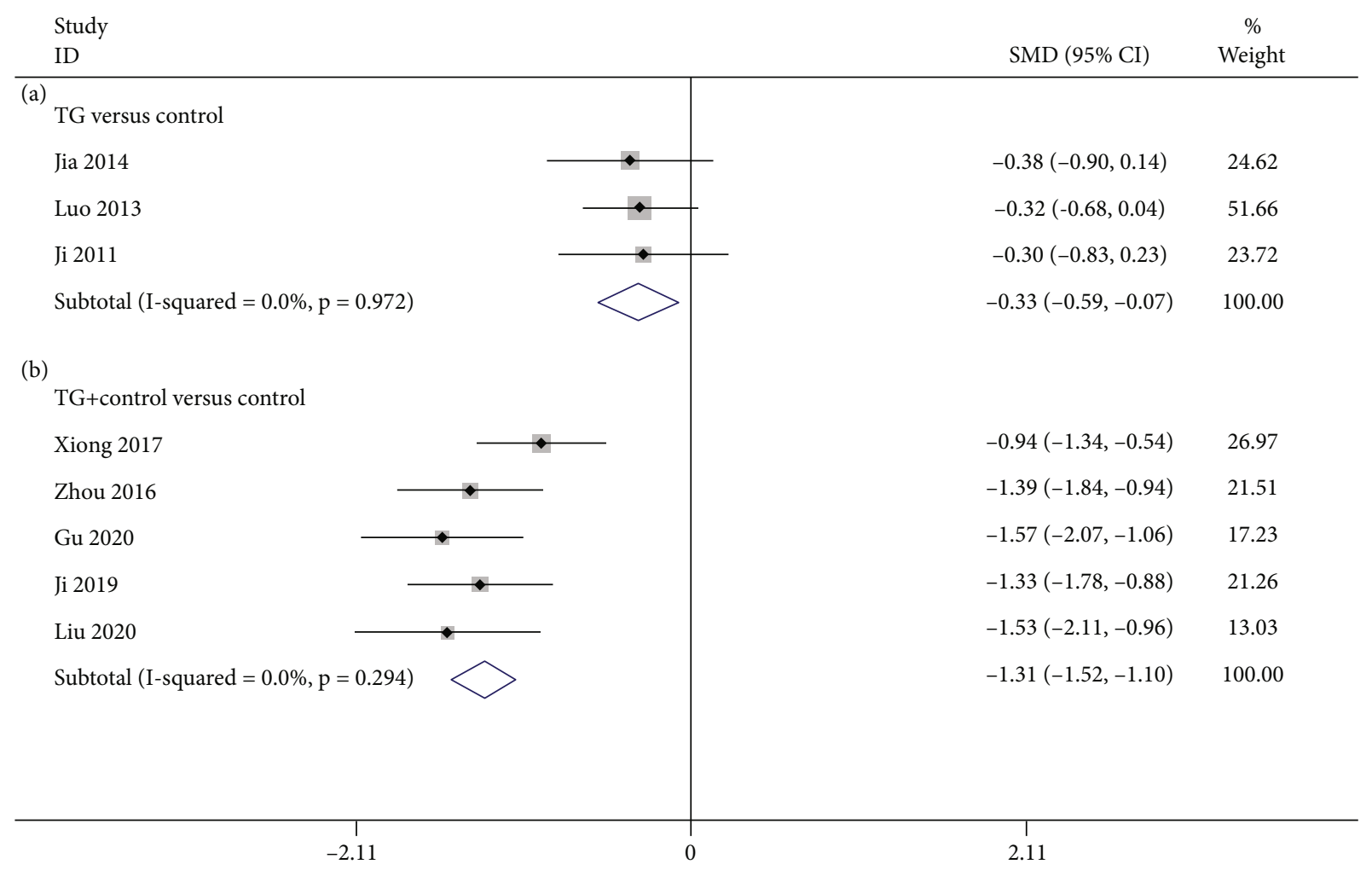

Figure 9: Forest plot of ESR: (a) TG versus control and (b) TG plus control versus control.

controlled trials are urgently needed. In the future, we recommend guidelines, covering CONSORT 2010 statement [43], that are needed to establish and report on randomized controlled trials of TG. Second, although TG treatment was found to be safe for AS patients in the analyzed studies, the safety of TG on AS remains to be further studied. The 


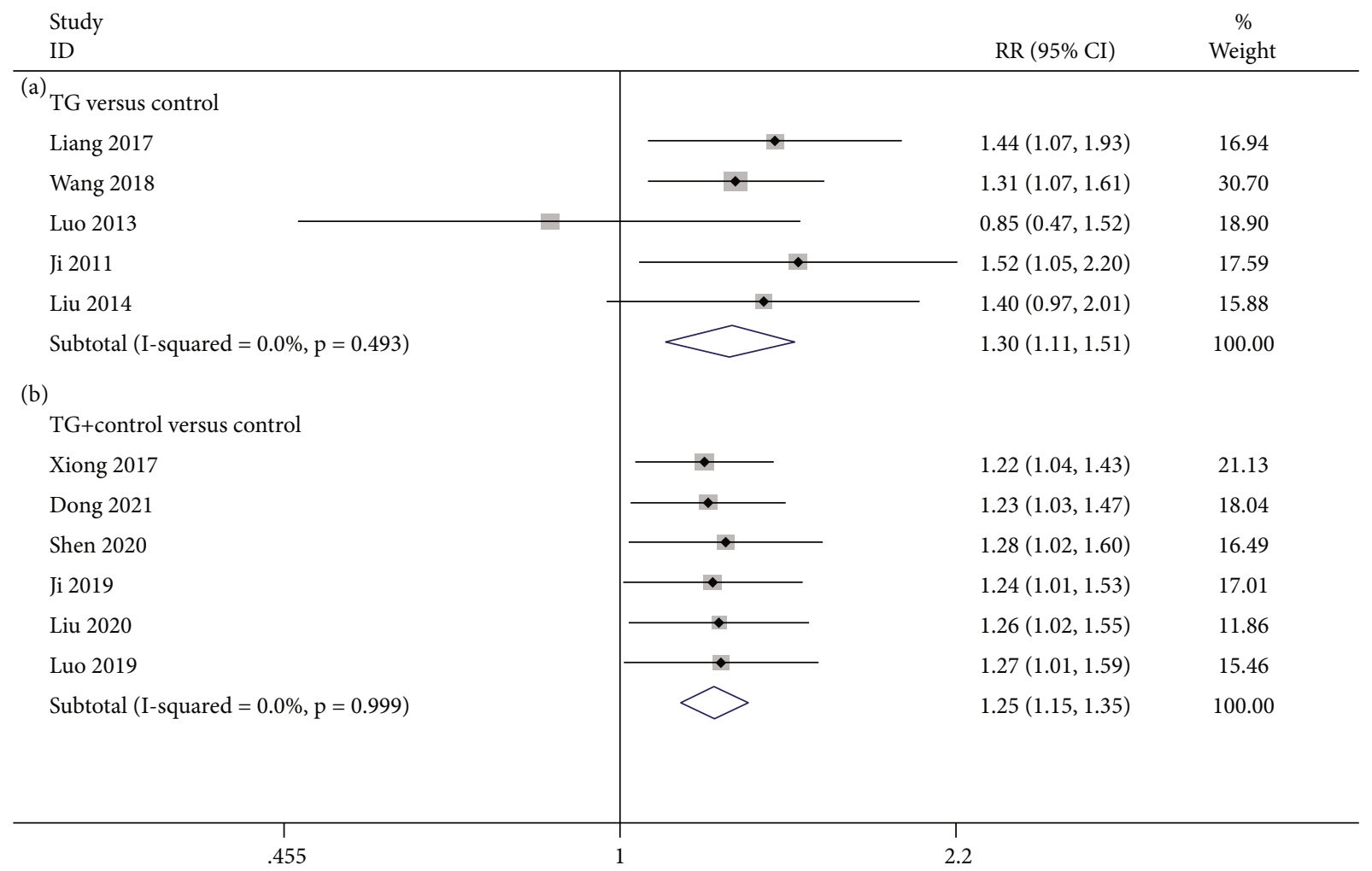

Figure 10: Forest plot of ER: (a) TG versus control and (b) TG plus control versus control.

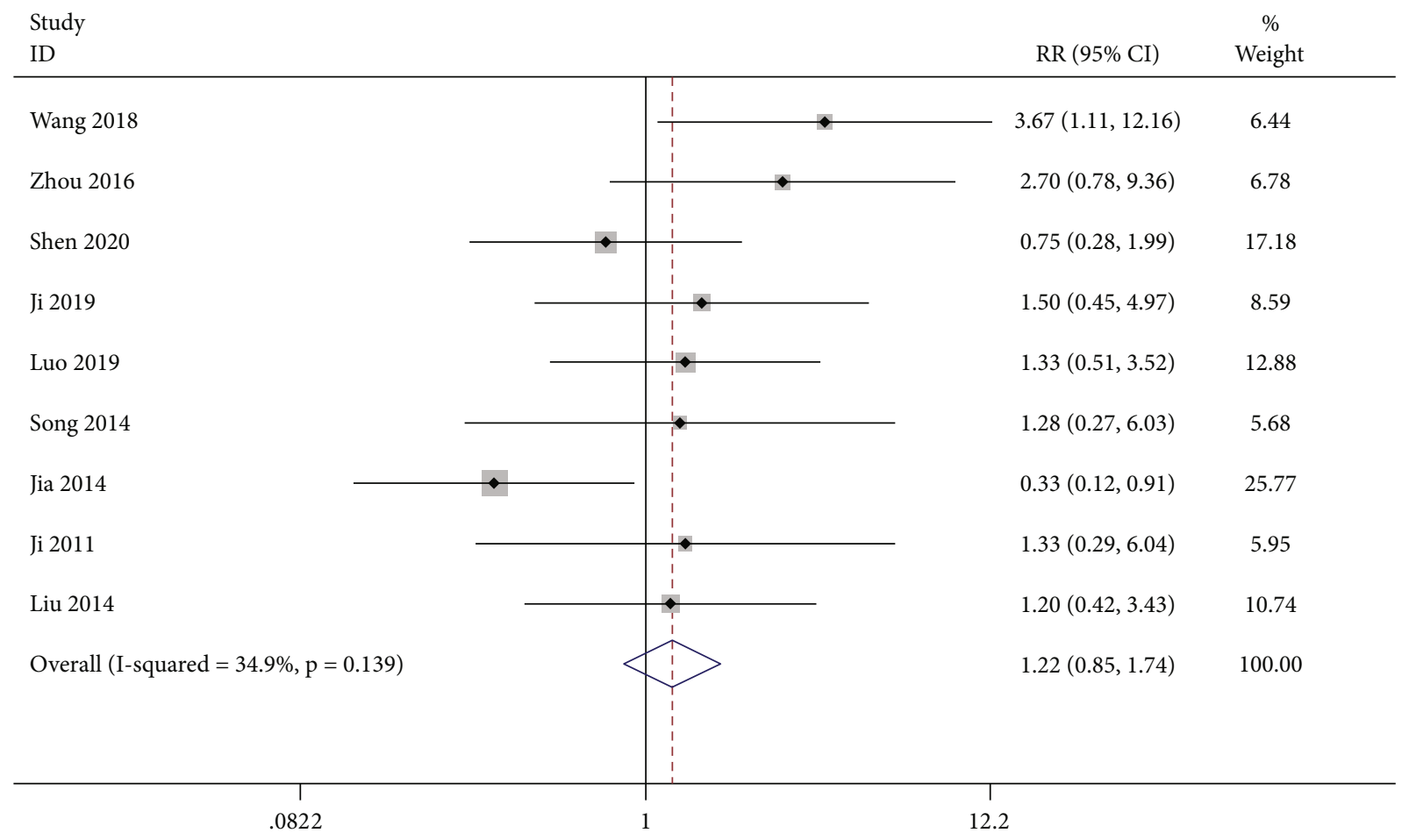

Figure 11: Forest plot of ADR.

standard reporting format of ADR was developed [44], and it was suggested to pay close attention to ADR reports of TG. Third, to finally understand the long-term safety of tri- glycerides in patients suffering from AS, clinical trials and studies with long follow-up periods are recommended. As indicated by the results of this paper, TG can be used as an 
TABLE 2: Subgroup analysis.

\begin{tabular}{|c|c|c|c|c|c|c|c|}
\hline Outcome & Subgroup factor & Number of study & Cases (EG/CG) & $I^{2}(\%)$ & Heterogeneity $(P)$ & Pooling model & $Z$ test $(P)$ \\
\hline \multirow[t]{17}{*}{ BASDAI } & & 8 & $336 / 296$ & 87.7 & $<0.0001$ & Random & $<0.0001$ \\
\hline & Course of treatment & & & & & & \\
\hline & $\leq 8$ weeks & 4 & $185 / 145$ & 55.3 & 0.082 & Random & $<0.0001$ \\
\hline & $>8$ weeks & 4 & $151 / 151$ & 75.6 & 0.006 & Random & $<0.0001$ \\
\hline & Sample size & & & & & & \\
\hline & $\leq 60$ & 3 & $98 / 79$ & 92.6 & $<0.0001$ & Random & 0.006 \\
\hline & $>60$ & 5 & $238 / 217$ & 80.4 & $<0.0001$ & Random & $<0.0001$ \\
\hline & Age & & & & & & \\
\hline & $\leq 35$ & 3 & $134 / 110$ & 94.0 & $<0.0001$ & Random & 0.042 \\
\hline & $>35$ & 5 & $202 / 186$ & 73.5 & 0.005 & Random & $<0.0001$ \\
\hline & Control medication & & & & & & \\
\hline & SSZ & 4 & $176 / 132$ & 75.8 & 0.006 & Random & $<0.0001$ \\
\hline & LEF & 2 & $89 / 90$ & 96.9 & $<0.0001$ & Random & 0.164 \\
\hline & ETA & 2 & $80 / 75$ & 84.3 & 0.012 & Random & 0.001 \\
\hline & TG dosage & & & & & & \\
\hline & $20 \mathrm{mg}$, tid & 5 & $223 / 175$ & 92.0 & $<0.0001$ & Random & 0.002 \\
\hline & $1 \mathrm{mg} / \mathrm{kg}$, tid & 3 & $113 / 121$ & 0 & 0.525 & Fixed & $<0.0001$ \\
\hline \multirow[t]{14}{*}{ SP-VAS } & & 5 & $224 / 195$ & 88.0 & $<0.0001$ & Random & 0.002 \\
\hline & Course of treatment & & & & & & \\
\hline & $\leq 8$ weeks & 4 & $179 / 150$ & 80.2 & 0.002 & Random & $<0.0001$ \\
\hline & $>8$ weeks & 1 & $45 / 45$ & - & - & Fixed & 0.824 \\
\hline & Sample size & & & & & & \\
\hline & $\leq 60$ & 2 & $53 / 53$ & 96.5 & $<0.0001$ & Random & 0.347 \\
\hline & $>60$ & 3 & $171 / 142$ & 45.5 & 0.160 & Fixed & $<0.0001$ \\
\hline & Age & & & & & & \\
\hline & $\leq 35$ & 3 & $150 / 126$ & 81.2 & 0.005 & Random & 0.086 \\
\hline & $>35$ & 2 & $74 / 69$ & 82.5 & 0.017 & Random & 0.001 \\
\hline & Control medication & & & & & & \\
\hline & SSZ & 2 & $69 / 44$ & 92.7 & $<0.0001$ & Random & 0.082 \\
\hline & LEF & 2 & $89 / 90$ & 90.6 & 0.001 & Random & 0.358 \\
\hline & ETA & 1 & $50 / 45$ & - & - & Fixed & $<0.0001$ \\
\hline
\end{tabular}

EG: experimental group; CG: control group; TG: Tripterygium glycosides; LEF: leflunomide; SSZ: sulfasalazine; ETA: etanercept; BASDAI: Bath Ankylosing Spondylitis Disease Activity Index; SP-VAS: Spinal Pain Visual Analog Score.

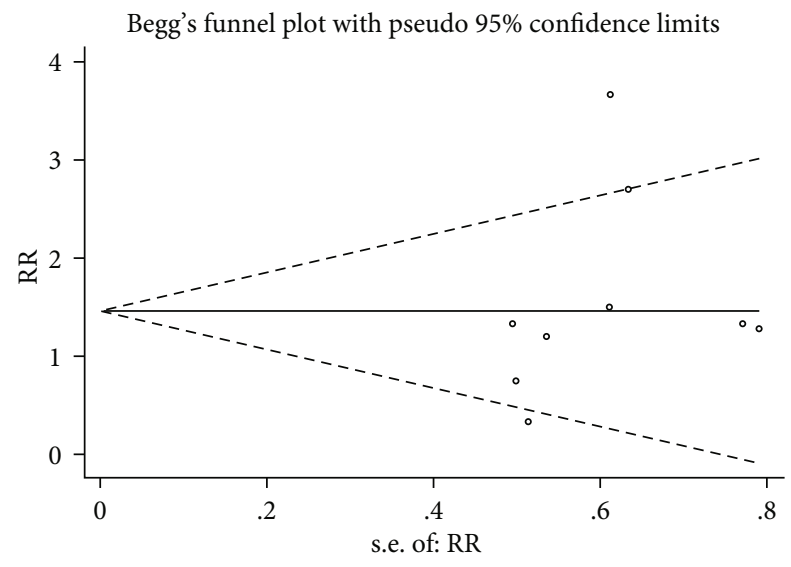

(a)

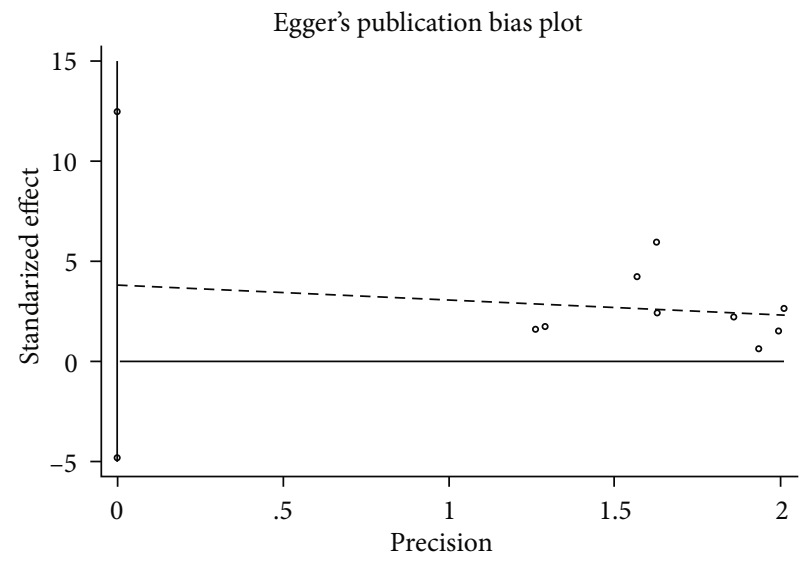

(b)

Figure 12: Begg's test and Egger's test of ADR. 

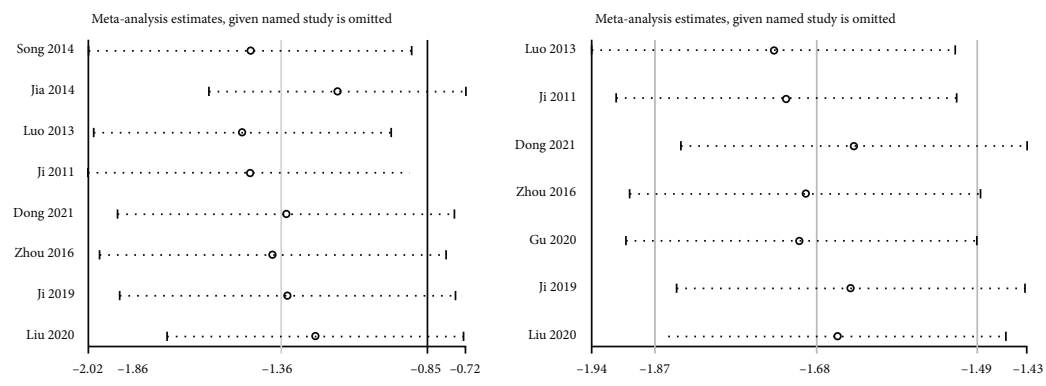

$$
\begin{array}{ll}
\text { | } & \text { Lower CI limit } \\
\text { ○ } & \text { Estimate } \\
\text { I } & \text { Upper CI limit }
\end{array}
$$

(a)

$$
\begin{array}{ll}
\text { Lower CI limit } \\
\text { - Estimate } \\
\text { | Upper CI limit }
\end{array}
$$

(b)
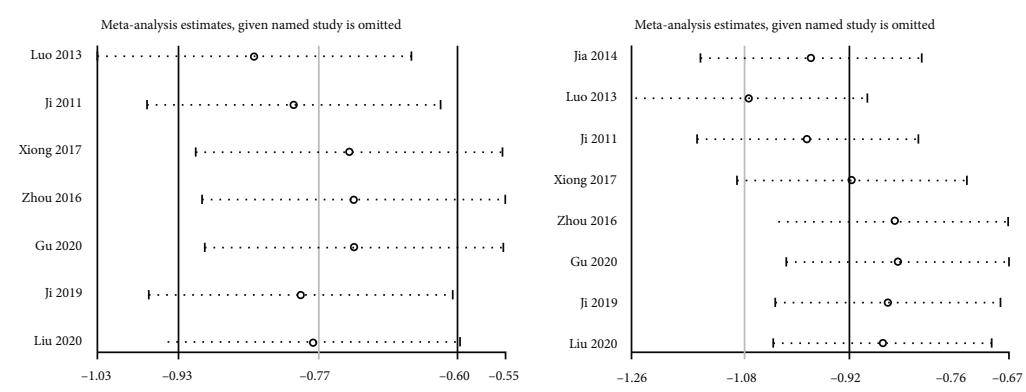

$$
\begin{array}{|l}
\text { Lower CI limit } \\
\text { - Estimate } \\
\text { | } \\
\text { Upper CI limit }
\end{array}
$$

(c)

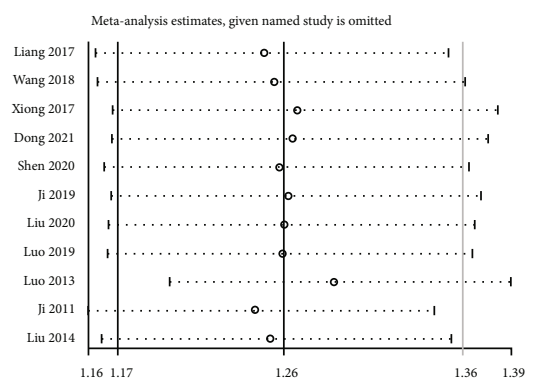

$$
\begin{array}{ll}
\mid & \text { Lower CI limit } \\
\text { - Estimate } \\
\text { | } & \text { Upper CI limit }
\end{array}
$$

(e)

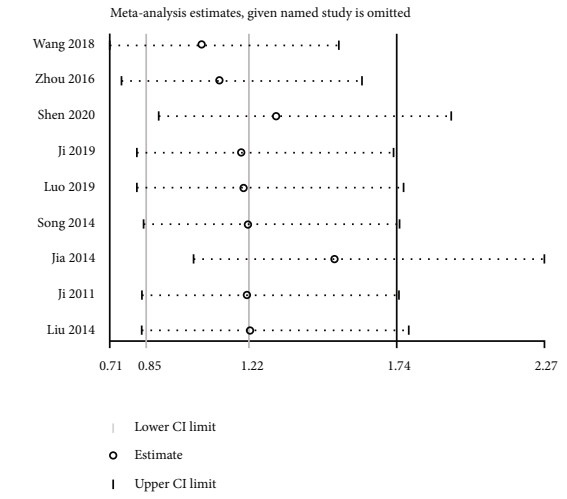

(f)

FIgURe 13: Sensitivity analysis for BASDAI (a), BASFI (b), CRP (c), ESR (d), ADR (e), and ER (f).

alternative treatment for patients suffering from AS, but further large-scale clinical studies are needed. In addition, the long-term safety, efficacy, and optimal dose of TG to treat AS should be explored.

4.6. Possible Mechanisms. Ji et al. [17] made a case-control investigation, reporting the ability of TG in improving the symptoms and signs of patients suffering from AS effectively on the basis of serum biomarker examination. The mechanism may be related to anti-inflammatory effects, inhibition of novel bone from being formed, and possible bone protective impact. Another study reported by them [45] showed that TG was efficient for the treatment of AS patients, and its mechanism of action may be correlated with the upregu- lation of cluster of differentiation (CD) $4^{+} \mathrm{CD} 25^{+} \mathrm{CD} 127^{\text {low }}$ regulatory $\mathrm{T}$ cells and the downregulation of interleukin(IL-) 17 levels in the peripheral blood. Furthermore, Zhang et al. [46] employed network pharmacology for the analysis of the active ingredients, the prediction of core TG targets, and pathways to treat AS, then preliminarily demonstrated the mentioned targets with the use of molecular docking. The enrichment investigation indicated that TG participates in various biological processes, covering acute inflammatory response, cell-matrix adhesion regulation, cell-cell adhesion, on the basis of TNF- $\alpha$, nuclear factor-kappa B (NF- $\kappa$ B), and other signal pathways. However, the specific mechanism should be further clarified further in vivo and in vitro tests are required. 


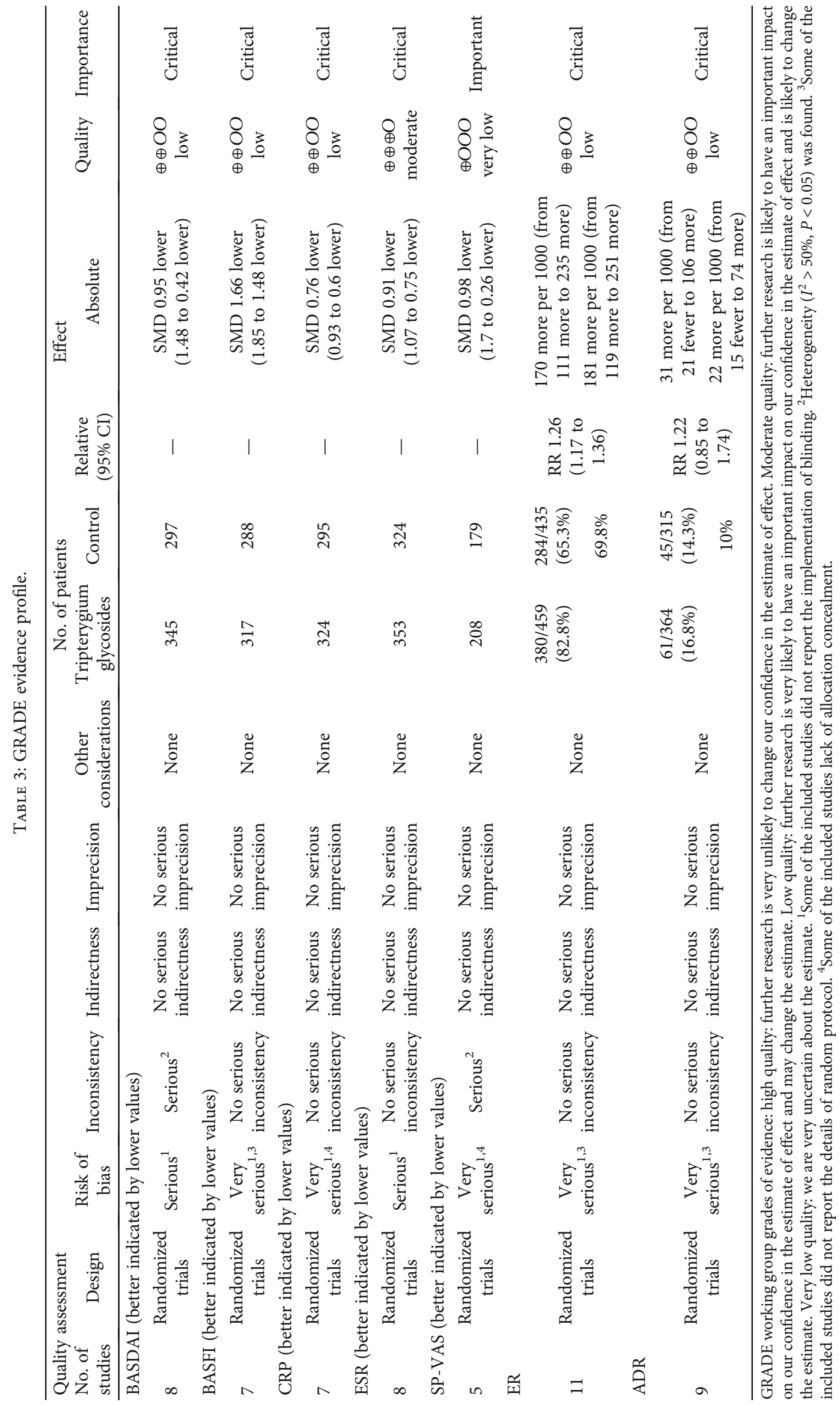




\section{Conclusion}

In brief, we showed supporting evidence that at least to an extent TG as adjuvant therapy or monotherapy for AS reduces the BASDAI, BASFI, SP-VAS, serum CRP, and ESR without increasing the ADR. Furthermore, TG treatment visibly improved the overall effective rate in AS. Therefore, the findings of this paper proved TG as a possible candidate in terms of AS treatment. Nevertheless, given the heterogeneity along with small sample size, more randomized controlled trials with large multicenter and high quality should be performed to deepen the benefits of TG to treat AS.

\section{Abbreviations}

$\begin{array}{ll}\text { AS: } & \text { Ankylosing spondylitis } \\ \text { TG: } & \text { Tripterygium glycosides } \\ \text { RCT: } & \text { Randomized controlled trial } \\ \text { ESR: } & \text { Erythrocyte sedimentation rate } \\ \text { CRP: } & \text { C-reactive protein } \\ \text { SP-VAS: } & \text { Spinal Pain Visual Analog Score } \\ \text { BASFI: } & \text { Bath Ankylosing Spondylitis Functional Index } \\ \text { BASDAI: } & \text { Bath Ankylosing Spondylitis Disease Activity } \\ & \text { Index } \\ \text { ADR: } & \text { Adverse drug reaction } \\ \text { HLA: } & \text { Human leukocyte antigen } \\ \text { TNF: } & \text { Tumor necrosis factor } \\ \text { DMARDs: } & \text { Disease-modifying antirheumatic drugs } \\ \text { NSAIDs: } & \text { Nonsteroidal anti-inflammatory drugs } \\ \text { SSZ: } & \text { Sulfasalazine } \\ \text { LEF: } & \text { Leflunomide } \\ \text { PRISMA: } & \text { Preferred Reporting Items in terms of System- } \\ & \text { atic Review and Meta-Analysis } \\ \text { AMSTAR: } & \text { Assessment of systematic review methodological } \\ & \text { quality } \\ \text { RR: } & \text { Relative risk } \\ \text { SMD: } & \text { Standard mean difference } \\ \text { ER: } & \text { Effective rate } \\ \text { RoB: } & \text { Risk of bias } \\ \text { CD: } & \text { Cluster of differentiation } \\ \text { IL: } & \text { Interleukin. } \\ & \end{array}$

\section{Data Availability}

Previously reported data were used to support this study. These prior studies and datasets are cited at relevant places within the text as references [24-38].

\section{Conflicts of Interest}

The authors disclose that this study was conducted without any commercial or financial relationships that could be construed as a prospective conflict of interest.

\section{Authors' Contributions}

Z.L. did the conceptualization, methodology, and software. J.R.C. did the data curation and writing of the initial draft. X.W.C. did the visualization and investigation. M.M.C. did the supervision. W.Z. and Z.L. did the software and validation. W.Z. did the writing-reviewing and editing.

\section{Acknowledgments}

The authors thank all the staff in the Department of Orthopaedic Surgery of The Dingli Clinical Institute of Wenzhou Medical University (Wenzhou Central Hospital).

\section{Supplementary Materials}

PRISMA_2020_checklist and search strategy were provided as supplementary material. (Supplementary Materials). (Supplementary Materials)

\section{References}

[1] A. S. Rolle, B. Zimmermann, and S. H. Poon, "Etanerceptinduced Henoch-Schönlein purpura in a patient with ankylosing spondylitis," Journal of Clinical Rheumatology, vol. 19, no. 2, pp. 90-93, 2013.

[2] J. Braun and J. Sieper, "Ankylosing spondylitis," The Lancet, vol. 369, no. 9570, pp. 1379-1390, 2007.

[3] H. Li, F. Guo, Y. C. Luo, J. P. Zhu, and J. L. Wang, "Efficacy of Tripterygium glycosides tablet in treating ankylosing spondylitis: a systematic review and meta-analysis of randomized controlled trials," Clinical Rheumatology, vol. 34, no. 11, pp. 1831-1838, 2015.

[4] J. J. de Winter, L. J. van Mens, D. van der Heijde, R. Landewe, and D. L. Baeten, "Prevalence of peripheral and extra-articular disease in ankylosing spondylitis versus non-radiographic axial spondyloarthritis: a meta-analysis," Arthritis Research \& Therapy, vol. 18, no. 1, p. 196, 2016.

[5] T. C. Shen, C. L. Lin, C. C. Wei et al., "The risk of asthma in patients with ankylosing spondylitis: a population-based cohort study," PLoS One, vol. 10, no. 2, article e0116608, 2015.

[6] A. Kanwal and S. Fazal, "Construction and analysis of proteinprotein interaction network correlated with ankylosing spondylitis," Gene, vol. 638, pp. 41-51, 2018.

[7] I. Sari, M. A. Ozturk, and N. Akkoc, "Treatment of ankylosing spondylitis," Turkish Journal of Medical Sciences, vol. 45, no. 2, pp. 416-430, 2015.

[8] I. H. Song, D. A. Poddubnyy, M. Rudwaleit, and J. Sieper, "Benefits and risks of ankylosing spondylitis treatment with nonsteroidal antiinflammatory drugs," Arthritis and Rheumatism, vol. 58, no. 4, pp. 929-938, 2008.

[9] J. Braun, R. van den Berg, X. Baraliakos et al., "2010 update of the ASAS/EULAR recommendations for the management of ankylosing spondylitis," Annals of the Rheumatic Diseases, vol. 70, no. 6, pp. 896-904, 2011.

[10] H. Haibel, F. Heldmann, J. Braun, J. Listing, H. Kupper, and J. Sieper, "Long-term efficacy of adalimumab after drug withdrawal and retreatment in patients with active nonradiographically evident axial spondyloarthritis who experience a flare," Arthritis \& Rheumatism, vol. 65, no. 8, pp. 2211-2213, 2013.

[11] Y. Xie, L. Tu, Y. Zhang et al., "Efficacy and safety of Fengshi Gutong Capsule in patients with active ankylosing spondylitis: a 4-week randomized controlled, double-blinded, doubledummy trial," Journal of Ethnopharmacology, vol. 285, article 114731, 2021. 
[12] A. Li, Y. Huang, S. Zheng, C. Tang, J. Zhu, and J. Li, "Clinical observation of Qushi Xiezhuo formula in reducing monosodium urate crystal deposition in patients with axial spondyloarthritis," Journal of Traditional Chinese Medicine, vol. 39, no. 5, pp. 722-729, 2019.

[13] M. Xue, Z. Z. Jiang, J. P. Liu et al., "Comparative study on the anti-inflammatory and immune suppressive effect of Wilforlide A," Fitoterapia, vol. 81, no. 8, pp. 1109-1112, 2010.

[14] C. Liu, Y. Zhang, X. Kong et al., "Triptolide prevents bone destruction in the collagen-induced arthritis model of rheumatoid arthritis by targeting RANKL/RANK/OPG signal pathway," Evidence-Based Complementary and Alternative Medicine, vol. 2013, Article ID 626038, 12 pages, 2013.

[15] G. X. Shui, Y. G. Wan, C. M. Jiang et al., "Progress in Tripterygium wilfordiiand its bioactive components in the field of pharmacodynamics and pharmacology," China Journal of Chinese Materia Medica, vol. 35, no. 4, pp. 515-520, 2010.

[16] M. X. Su, W. D. Zhou, J. Lan, B. Di, and T. J. Hang, "Rapid and sensitive analysis of multiple bioactive constituents in Tripterygium glycosides tablets using liquid chromatography coupled with time-of-flight mass spectrometry," Journal of Separation Science, vol. 38, no. 5, pp. 804-812, 2015.

[17] W. Ji, Y. Chen, X. Zhao et al., "Beneficial effects of Tripterygium glycosides tablet on biomarkers in patients with ankylosing spondylitis," Molecular Medicine Reports, vol. 12, no. 1, pp. 684-690, 2015.

[18] D. Moher, A. Liberati, J. Tetzlaff, D. G. Altman, and for the PRISMA Group, "Preferred reporting items for systematic reviews and meta-analyses: the PRISMA statement," BMJ, vol. 339, article b2535, 2009.

[19] J. Chen, J. Zheng, M. Chen, S. Lin, and Z. Lin, "The efficacy and safety of Chinese herbal medicine Xianling Gubao capsule combined with alendronate in the treatment of primary osteoporosis: a systematic review and meta-analysis of 20 randomized controlled trials," Frontiers in Pharmacology, vol. 12, article 695832, 2021.

[20] J. Sieper, M. Rudwaleit, X. Baraliakos et al., "The Assessment of SpondyloArthritis international Society (ASAS) handbook: a guide to assess spondyloarthritis," Annals of the Rheumatic Diseases, vol. 68, Supplement 2, pp. ii1-ii44, 2009.

[21] J. Higgins, "Cochrane Handbook for Systematic Reviews of Interventions Version 5.1.0," in Naunyn-Schmiedebergs Archiv für experimentelle Pathologie und Pharmakologie, p. S38, The Cochrane Collaboration, 2011.

[22] H. Balshem, M. Helfand, H. J. Schünemann et al., "GRADE guidelines: 3 . Rating the quality of evidence," Journal of Clinical Epidemiology, vol. 64, no. 4, pp. 401-406, 2011.

[23] G. H. Guyatt, A. D. Oxman, S. Sultan et al., "GRADE guidelines: 9. Rating up the quality of evidence," Journal of Clinical Epidemiology, vol. 64, no. 12, pp. 1311-1316, 2011.

[24] C. C. Luo, "Clinical controlled trial study of leflunomide and Tripterygium glycosides in the treatment of ankylosing spondylitis," Chinese Journal of Primary Medicine and Pharmacy, vol. 20, no. 11, pp. 1716-1718, 2013.

[25] Z. G. Liang, "The clinical effect of Tripterygium glycosides in the treatment of ankylosing spondylitis," China Practical Medicine, vol. 12, no. 1, pp. 136-137, 2017.

[26] Q. Zhou, "Clinical efficacy and safety of Tripterygium glycosides combined with etanercept in the treatment of ankylosing spondylitis," The Journal of Medical Theory and Practice, vol. 29, no. 6, pp. 763-764, 2016.
[27] Q. W. Luo, G. Q. Chen, H. W. Zhang et al., "Clinical trial of etanercept injection combined with Tripterygium glycosides tablets in the treatment of ankylosing spondylitis," The Chinese Journal of Clinical Pharmacology, vol. 35, no. 15, pp. 1578-1580, 2019.

[28] K. Shen, X. R. Wang, Q. C. Hua, and Z. L. Li, "Clinical trial of Tripterygium glycoside tablets combined with etanercept in the treatment of patients with ankylosing spondylitis," The Chinese Journal of Clinical Pharmacology, vol. 36, no. 19, pp. 2996-2999, 2020.

[29] T. M. Jia and W. J. Li, "The contrastive research about the effect and safety of leflunomide and tripterysium glycosides on treating ankylosing spondylitis," Medical Recapitulate, vol. 20, no. 19, pp. 3612-3613, 2014.

[30] Y. Liu, "Effect of etanercept solution for injection combined with Tripterygium glycosides tablets in the treatment of ankylosing spondylitis," China Practical Medicine, vol. 15, no. 36, pp. 109-111, 2020.

[31] C. Y. Gu, "Effect of Tripterygium glycoside tablets in adjuvant treatment of ankylosing spondylitis," Modern Diagnosis and Treatment, vol. 31, no. 9, pp. 1368-1370, 2020.

[32] J. W. Xiong and J. F. Chen, "Effects of Tripterygium glycosides on transforming growth factor $\beta$ and anxiety in patients with ankylosing spondylitis," Henan Traditional Chinese Medicine, vol. 37, no. 1, pp. 119-121, 2017.

[33] S. F. Liu, B. P. Zhang, H. Wang, and X. Tan, "Efficacy and safety of Tripterygium glycosides in the treatment of ankylosing spondylitis," Journal of North Pharmacy, vol. 11, no. 6, pp. 91-93, 2014.

[34] Y. X. Dong, "Efficacy of Tripterygium glycosides combined with sulfasalazine in the treatment of ankylosing spondylitis," Practical Clinical Journal of Integrated Traditional Chinese and Western Medicine, vol. 21, no. 17, pp. 129-130, 2021.

[35] M. J. Wang, "Evaluation of efficacy and safety of Tripterygium glycosides in the treatment of ankylosing spondylitis," Journal of Clinical Medical, vol. 5, no. 18, p. 84, 2018.

[36] Y. N. Song and W. Ji, "Evaluation of Tripterygium glycosides with ASDAS-CRP in the treatment of 39 cases of ankylosing spondylitis," Medical Journal of Communications, vol. 28, no. 4, pp. 366-370, 2014.

[37] W. Ji, Y. N. Song, H. G. Li, and C. G. Ma, “Therapeutic effect of Tripterygium glycosides tablet on 45 cases of ankylosing spondylitis," Shandong Medical Journal, vol. 51, no. 47, pp. 76-77, 2011.

[38] J. H. Ji and J. Cheng, “Therapeutic effect of Tripterygium wilfordii polyglycosides on patients with ankylosing spondylitis and its effect on soluble interleukin-2 receptor levels," Hainan Medical Journal, vol. 30, no. 22, pp. 2905-2908, 2019.

[39] T. R. Jenkinson, P. A. Mallorie, H. C. Whitelock, L. G. Kennedy, S. L. Garrett, and A. Calin, "Defining spinal mobility in ankylosing spondylitis (AS). The Bath AS Metrology Index," The Journal of Rheumatology, vol. 21, no. 9, pp. 1694-1698, 1994.

[40] S. van der Linden and D. van der Heijde, "Ankylosing spondylitis: clinical features," Rheumatic Disease Clinics of North America, vol. 24, no. 4, pp. 663-676, 1998.

[41] G. Murdaca, B. M. Colombo, and F. Puppo, "Anti-TNF-alpha inhibitors: a new therapeutic approach for inflammatory immune-mediated diseases: an update upon efficacy and adverse events," International Journal of Immunopathology and Pharmacology, vol. 22, no. 3, pp. 557-565, 2009. 
[42] Y. Li, T. L. Shi, Y. H. Zhang et al., "Meta-analysis on efficacy and safety of Tripterygium glycosides tablets in treatment of ankylosing spondylitis," Evaluation and Analysis of Drug-Use in Hospitals of China, vol. 16, no. 12, pp. 1680-1685, 2016.

[43] C. W. Cheng, T. X. Wu, H. C. Shang et al., "CONSORT extension for Chinese herbal medicine formulas 2017: recommendations, explanation, and elaboration," Annals of Internal Medicine, vol. 167, no. 2, pp. 112-121, 2017.

[44] Z. X. Bian, H. Y. Tian, L. Gao et al., "Improving reporting of adverse events and adverse drug reactions following injections of Chinese materia medica," Journal of Evidence-Based Medicine, vol. 3, no. 1, pp. 5-10, 2010.

[45] W. Ji, H. Li, F. Gao, Y. Chen, L. Zhong, and D. Wang, "Effects of Tripterygium glycosides on interleukin-17 and $\mathrm{CD}^{+-}$ $\mathrm{CD} 25^{+} \mathrm{CD} 127$ low regulatory $\mathrm{T}$-cell expression in the peripheral blood of patients with ankylosing spondylitis," Biomedical Reports, vol. 2, no. 4, pp. 517-520, 2014.

[46] J. Zhang, Y. Zhou, and Z. Ma, "Multi-target mechanism of $<\mathrm{i}>$ Tripteryguim wilfordii $</ \mathrm{i}>$ hook for treatment of ankylosing spondylitis based on network pharmacology and molecular docking," Annals of Medicine, vol. 53, no. 1, pp. 1090$1098,2021$. 